\title{
Rosetta and 67P/Churyumov-Gerasimenko: a comet under observation
}

\author{
Gabriele E. Arnold*, \\ *Deutsches Zentrum für Luft- und Raumfahrt e.V. (DLR), Institute of Planetary Research, \\ Rutherfordstrasse 2, 12489 Berlin, Germany. \\ *Gabriele.Arnold@dlr.de; phone +49-3067055370; fax + 49-3067055303
}

\begin{abstract}
After a ten year's expedition through the Solar system Europe's comet chaser Rosetta arrived at comet 67 P/ChuryumovGerasimenko in August 2014. Less than 100 kilometers from the nucleus the eleven orbiter payload instruments started to map and characterize the comet in great detail. In November 2014 Philae was the first robotic subsystem ever that landed on a cometary surface performing in situ measurements with ten instruments. The mission's scientific program following the deployment of Philae is determined by the activity of the comet, which will increase as 67P approached perihelion in August 2015. This paper is a review article. It introduces the mission goals and profile. It gives an overview of some of the preliminary results of the mission. Selected results gained during the pre-landing phase of the subsystem Philae and the comet's escort phases are discussed.
\end{abstract}

Keywords: Planetary remote sensing, comets, Rosetta.

\section{INTRODUCTION}

Comets are the most primitive objects in the solar system. They can be considered as some of the remaining original elements in our 4.6 billion-years-old solar system. These minor bodies were formed in the outer and cold regions of the planetary system, and thus they are less altered containing information about the early solar nebula's state of matter ${ }^{1,2}$. The precursor cometary material may be a combination of unaltered interstellar grains and volatiles that would be subsequently condensed in the protoplanetary disk. ${ }^{1}$ Therefore, comets represent material closest to the conditions of the early solar system. Previous spacecraft observations of comets with Giotto (1P/Halley $)^{3,4,5}$, Stardust (81P/Wild 2) ${ }^{6}$, Deep Impact (9P/Tempel 1) $)^{7}$, and EPOXI (103P/Hartley 2$)^{8}$ provided insights into cometary structures and first close-up views of cometary nuclei. Nevertheless, all of these space missions have flown by their targets, and thus they took only timelimited snapshots of comets within their orbital evolutions.

ESA's cornerstone Rosetta to comet 67P/Churyumov-Gerasimenko is the first mission escorting a comet during its passage through the inner solar system. Additionally, a robotic device, "Philae", which landed on the cometary surface in November 2014, provided important in situ data. ESA's Rosetta mission was launched on March 2, 2004 on an Ariane 5 G+ rocket. It rendezvoused with comet 67P/Churyumov-Gerasimenko, henceforth shortly called 67P, in May 2014 starting its mapping campaign in August 2014 ${ }^{9}$.

Rosetta consists of an orbiter and the lander system. The Rosetta orbiter's scientific payload includes eleven scientific instruments devoted to study the cometary nucleus and coma within the orbital evolution of 67P. The Philae lander houses ten scientific instruments for cometary in situ analyses ${ }^{9}$. During its first year of observation Rosetta performed unique investigations for periods of global mapping, lander deployment, and comet chase toward the Sun.

This paper aims to place Rosetta in context to previous space borne cometary missions. It summarizes the mission's key goals, the mission's profile, and some of the mission highlight results. The paper bases on recent results published by the different Rosetta instrument teams, whose specific scientific achievements were documented and carefully quoted.

Infrared Remote Sensing and Instrumentation XXIII, edited by Marija Strojnik Scholl,

Gonzalo Páez, Proc. of SPIE Vol. 9608, 960802 - (c) 2015 SPIE

CCC code: $0277-786 \mathrm{X} / 15 / \$ 18 \cdot$ doi: $10.1117 / 12.2190445$

Proc. of SPIE Vol. $9608960802-1$ 


\section{ROSETTA MISSION}

The Rosetta mission is a cooperative project between ESA, various European national space agencies, and NASA, and is comprised of eleven orbital and ten lander experiments, making it unprecedented in scale. Rosetta rendezvoused the comet 67P in May 2014 at 4 AU from the Sun. After a 10-years cruise, the Rosetta spacecraft began a close exploration of comet 67P in July 2014 and entered a 30-km bound orbit around the comet on September 9, 2014. It will accompany the comet's journey towards perihelion (August 13, 2015, 1.24 AU). ESA's cornerstone Rosetta is the first spacecraft to orbit a comet and was the first to land a robotic device on the surface of a comet. Therefore, Rosetta combines remote sensing and in situ investigations.
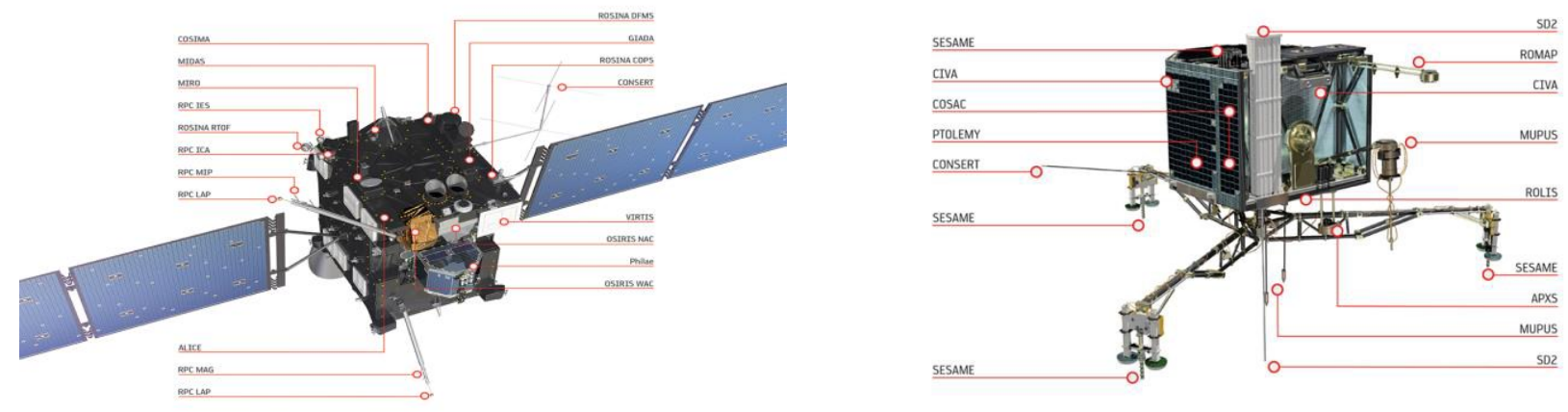

Figure 1. Rosetta Spacecraft: Left - Orbiter; credit: ESA, right - Philae lander; credits: ESA, DLR (Description of the instrument's acronyms is given in section 2.3).

\subsection{Rosetta's goals}

The mission aims at a global characterization of the cometary nucleus, the determination of dynamic properties, the surface morphology and composition, chemical /mineralogical /isotopic compositions of volatiles and refractories, physical properties, studies of cometary activity, and evolution of interaction with the solar wind ${ }^{9}$. The primary mission goals are:

- Perform a extended exploration of a comet at close quarters to observe how it is transformed by the warmth of the Sun along its elliptical orbit ${ }^{10}$,

- Land a probe on a comet's nucleus for in-situ analyses ${ }^{10}$,

- Observe the comet's nucleus (shape, dynamic properties, surface, and interior) and coma from close range,

- Analyse the chemical, mineralogical, and isotopic composition of the nucleus (volatiles and refractories),

- Study the physical properties of the cometary nucleus,

- Observe the comet's activity as it approaches the Sun and study its interaction with the environment,

- Investigate the dynamic coma structure and composition (structure, thermal, electrical, and magnetic properties),

- Study changes related to heliocentric distance, cometary, and seasons.

This will enable to investigate how the solar system evolved to its current state:

- How did comets originate and where have they been formed?

- What is the relationship of their materials to those found in other regions of the solar system and in interstellar space?

- How have comets contributed to the origin and evolution of planets, moons, and other minor bodies (relationship to asteroids) in the solar system?

- Which role do they play in feeding the inner solar system with volatiles?

To meet these goals the Rosetta mission was designed to rendezvous 67P in the cold outer region of the solar system when the comet was less active, orbit the cometary nucleus, examine it from close proximity, fly alongside the comet 
towards the inner solar system, and land a robotic device on its surface. A sophisticated orbiter scientific payload consisting of optical, microwave, and radar systems, gas/dust analyzers, plasma/magnetic field and radio science instruments was selected to carry out the close-up remote observations ${ }^{11}$. Philae's scientific instruments were aimed to perform multispectral and multicolor imaging at the comet's surface, study elemental composition, thermal, mechanical, electrical, and magnetic properties ${ }^{12}$.

\subsection{Rosetta's journey}

Rosetta launched on 2 March 2004 by an Ariane-5 G+ from Kourou, French Guiana. During its ten year's journey until arrival at 67P the spacecraft received four gravity assist manoeuvers from Earth (March 2005, November 2007, and November 2009) and from Mars (February 2007) ${ }^{13}$. Passing the main asteroid belt Rosetta studied two asteroids flown by, 2867 Šteins on September 2008 and 21 Lutetia on July 2010. After the hibernation phase, which started in June 2011, Rosetta was waking up in January 2014 and rendezvoused 67P in May 2014. From August to November 2014 an extensive pre-landing observation phase followed to map the comet and select a landing site for Philae. Currently Rosetta is escorting 67P to collect comprehensive data, while the comet becomes progressively active. 67P passed the perihelion in August 13, 2015 and was tracked as the comet moves again away from the Sun.

\subsection{Rosetta's scientific payload}

Rosetta's orbital payload (see Fig. 1, right) consists of eleven scientific instruments. Among the optical systems it houses the OSIRIS Optical, Spectroscopic, and Infrared Remote Imaging System) camera ${ }^{14}$ the VIRTIS (Visible and Infrared Thermal Imaging Spectrometer) ${ }^{15,16,17}$ and the ALICE (miniaturized ultraviolet imaging spectrometer) ${ }^{18}$ spectrometers to observe 67P in the UV to IR spectral range. The microwave instrument MIRO (Microwave Instrument for the Rosetta Orbiter) $^{19}$ aims to study minor gases and the nucleus' subsurface temperature. CONSERT (Comet Nucleus Sounding Experiment by Radiowave Transmission $)^{20,38}$ is the radar sounding device to investigate the comet's internal structure. Gas and dust analyzers like ROSINA (Rosetta Orbiter Spectrometer for Ion and Neutral Analysis) ${ }^{21}$, COSIMA (Cometary Secondary Ion Mass Analyser) $^{22}$, GIADA (Grain Impact Analyser and Dust Accumulator) ${ }^{23}$, and MIDAS (Micro-Imaging Dust Analysis System) ${ }^{24}$ are dedicated to study the structure, composition, and dynamics of gaseous and dust material in the cometary coma. The Rosetta Plasma Consortium (RPC) ${ }^{25}$ and the Rosetta Radio Science Investigations (RSI) ${ }^{26}$ complete the orbiter payload aiming to measure the nucleus' physical properties, the comet's interaction with the solar wind, and to determine mass and gravity of the comet, respectively.

Philae's payload (see Fig. 1, right) combines a set of ten instruments to image, analyze, and study the comet by in situ measurements. The cameras CIVA (Comet Infrared and Visible Imager) ${ }^{27}$ and ROLIS (Rosetta Lander Imaging System $)^{28}$ performed multispectral, color, and panchromatic imaging on the immediate cometary surface. COSAC (Cometary Sampling and Composition) is an instrument consisting of a gas chromatograph and a time-of-flight mass spectrometer $(\mathrm{MS})^{29}$. It enables to analyze samples delivered by a drilling device and can operate in a sniffing mode as well as using $\mathrm{MS}^{30}$. The PTOLEMY ${ }^{31}$ instrument is designed to measure stable isotope ratios and elements such as $\mathrm{H}, \mathrm{C}$, $\mathrm{N}$, and $\mathrm{O}$ of samples procured from the drilling device ${ }^{32}$. The instrument concept is termed MODULUS (Methods Of Determining and Understanding Light elements from Unequivocal Stable isotope compositions) ${ }^{33}$. A further experiment to study chemical composition is APEX (Alpha Proton X-ray Spectrometer), which has not been active so far ${ }^{34}$. MUPUS (Multipurpose Sensors for Surface and Sub-Surface Science) ${ }^{35,36}$ and SESAME (Surface Electrical Sounding and Acoustic Monitoring Experiment) ${ }^{37}$ are designed to investigate physical properties of the surface and sub-surface like temperature, thermal inertia, electrical conductivity, and mechanical characteristics. The Comet Nucleus Sounding Experiment by Radiowave Transmission (CONSERT) is a bi-static radar instrument, which propagates long-wavelength signals between the Rosetta orbiter and the lander Philae revealing information about the permittivity of the nucleus, which depends on porosity, composition, and temperature and thus providing insights into its internal structure ${ }^{20,38}$. The magnetometer and plasma monitor, ROMAP (Rosetta Magnetometer and Plasma Monitor) ${ }^{39}$, is dedicated to study magnetic field and plasma properties, and the drilling device SD2 (Sample and Distribution Device) was aiming to collect and deliver samples to different ovens or microscope inspection ${ }^{40}$.

Originally to by touched down the nucleus' region called Agilkia (see Fig. 2) Philae rebounded twice because the harpoon anchors to fire and gas repulsion mechanism failed, before finally landing at Abydos. Due to the unfavorable illumination conditions at the final landing site, the lander's solar arrays have not been illuminated sufficiently to reload its batteries for long duration measurements. Nevertheless, Philae was operating about sixty hours on the surface of the 
comet in November 2014. Most of the experiments could collect in situ data. With the increase of solar insolation the contact to Philae could be reactivated in June 2015. Currently the future operation of Philae and its instruments is under investigation.

\section{ROSETTA'S OBSERVATION RESULTS}

The Jupiter Family Comet 67P discovered in 1969 is characterized by an eccentricity of 0.64102, an inclination of $7.0405^{\circ}$, a perihelion distance of 1.2432 AU, and a semi-major axis of 3.4630 AU resulting in an orbital period of 6.44 years. In $195967 \mathrm{P}$ had a close encounter with Jupiter which changed its perihelion distance from 2.74 AU to its present value $^{41}$. The current rotation period is about $12.4 \mathrm{~h}^{42}$. During the pre-landing (before November 12, 2014) and escort phase (after November 12, 2014), the orbiter instruments collected data to characterize the comet with remote sensing optical systems, with a microwave device, with radar sounding, gas and dust analyses, plasma and magnetic field studies, and radio sounding 43,44, 45, 46, 47, 48, 49 . First evaluation of Philae's in situ measurements revealed information about the chemical composition of the comet's gas and dust ${ }^{30,32}$. Complex organic molecules have been detected ${ }^{32}$, high resolution close-up images from the landing sites have been taken ${ }^{50,51}$, and physical-mechanical properties of cometary material have been determined ${ }^{36,38,39}$.

\subsection{Orbiter observations}

Cometary nucleus mapping and physical properties: Rosetta's OSIRIS camera mapped the comet disclosing a structure of two lobes connected by a neck (see Fig. 2) ${ }^{43}$. The cometary surface displays different morphologies including dust covered terrains (see Fig. 2, middle: see e.g. the region Ash), brittle material (see e.g. Seth), large scale depressions (see e.g. Aten), smooth terrains and exposed consolidated surfaces (see e.g. Aker; see also Fig. 1, reference 17, 9608-2, these proceedings) $)^{44}$.
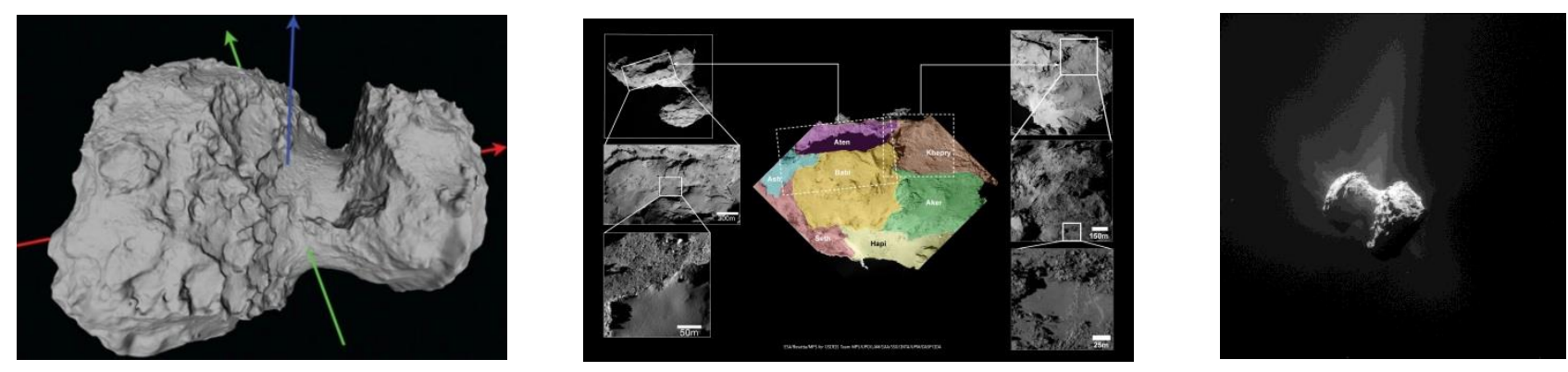

Figure 2. OSIRIS results. Left: shape model of 67P with rotation axis (blue) and equatorial $\mathrm{x}$ and y axis (red, green) ${ }^{43}$. Credit: ESA/Rosetta/MPS for OSIRIS Team MPS/UPD/LAM/IAA/SSO/INTA/UPM/DASP/IDA.

Middle: Cometary surface details: Context image of 67P's surface features close to regional boundaries on comet's large lobe. Credit: ESA/Rosetta/MPS for OSIRIS Team MPS/UPD/LAM/IAA/SSO/INTA/UPM/DASP/IDA ${ }^{52}$. Right: Observation of cometary activity (Rosetta NAVCAM), July 20, 2015. Credits: ESA/Rosetta/NAVCAM - CC BY-SA IGO 3.0.

The larger lobe (body) has a size ${ }^{43}$ of about $4.1 \mathrm{~km} \times 3.3 \mathrm{~km} \times 1.8 \mathrm{~km}$, and the smaller one $(\text { head) })^{43}$ is $2.6 \mathrm{~km} \times 2.3 \mathrm{~km}$ $\times 1.8 \mathrm{~km}$. The preliminary calculated total volume is about $21.4 \pm 2.0 \mathrm{~km}^{3}$. With a mass of $1.0 \times 10^{13} \mathrm{~kg}$ determined by $\mathrm{RSI}^{55}$ the resulting mean density is $470 \pm 45 \mathrm{~kg} / \mathrm{m}^{3}\left(\mathrm{see}^{43}\right)$. This low density is comparable with cork and is an indication of high porosity of $70-80 \% .^{43}$ The gravitational potential varies by less than a factor of two ${ }^{43}$. The obliquity of the comet's rotation axis is $52^{\circ}$ causing cometary seasons. Therefore, during the pre-landing, landing, and early escort phases only the northern hemisphere of 67P was illuminated. As the comet approaches its perihelion the southern hemisphere is progressively illuminated, enabling to disclose the southern part of 67P.

$67 \mathrm{P}$ has a low average normal albedo ${ }^{49}$ of $0.060 \pm 0.003$ and a geometric albedo ${ }^{53}$ of $\mathrm{A}_{\text {geo }}=0.062 \pm 0.002$ at $550 \mathrm{~nm}$. The analysis of the nucleus' surface composition by VIRTIS shows that the surface is depleted by volatiles. VIRTIS close-up observations revealed only very limited amounts of isolated ice patches. A broad absorption feature in the 2.9-to 3.6- $\mu$ m 
range across the entire illuminated surface was detected. This feature is assigned to nonvolatile organic macromolecular materials combining $\mathrm{CH}, \mathrm{C}-\mathrm{OH},-\mathrm{COOH}$, and $\mathrm{NH}$ chemical groups ${ }^{49,17}$. Measurements of the surface ${ }^{49}$ and sub-surface temperatures ${ }^{45}$ enable to study the upper layered nucleus structure and its thermo-electrical properties.

OSIRIS, NAVCAM (see Fig. 2, right), VIRTIS, ROSINA, MIRO, and other orbital instruments detected the presence of a gaseous dusty coma increasingly intensifying as the comet approached its perihelion distance to the Sun. Early activity was first observed over the neck (Hapi) region.

Gas and dust observations: Very early water vapor was detected by MIRO in June 2014, when the comet was at $3.92 \mathrm{AU}$ from the Sun. The mean total water production rate over one nucleus rotation was $1 \times 10^{25}$ molecules $/ \mathrm{s}(300 \mathrm{ml} / \mathrm{s})$ in June $2015^{45}$. VIRTIS-H measurements were performed to study the productions and distributions of water $(2.67 \mu \mathrm{m}$ signature) and carbon dioxide $(4.27 \mu \mathrm{m})$ in the cometary coma, when the comet was between 2.47 and 2.91 AU from the Sun resulting in a water production rate of $6.1 \times 10^{25}$ to $1.0 \times 10^{26}$ molecules/s during the 12 -h nucleus rotation ${ }^{54}$. The water vapor production rate decreases for low solar illumination, whereas $\mathrm{CO}_{2}$ is outgassing from both illuminated and non-illuminated areas ${ }^{54}$. This distribution of the two species results in variable $\mathrm{CO}_{2} / \mathrm{H}_{2} \mathrm{O}$ column density ratios between $2 \%$ and $30 \% .{ }^{54}$ Rotational temperatures have been used to determine coma temperatures of typically $100 \mathrm{~K}$ above $1 \mathrm{~km}$ from the nucleus ${ }^{54}$. ROSINA measured the gaseous coma composition in August and September 2014 ${ }^{46}$. Water, Carbon monoxide, and carbon dioxide have been identified. The measurements show large abundance fluctuations related to diurnal and seasonal variations and a coma composition being highly heterogenous ${ }^{46}$. The highest fluctuations occur for water vapor. Smaller abundance variations of $\mathrm{CO}_{2}$ and $\mathrm{CO}$ may indicate that they sublimate from grater depths ${ }^{46}$. Finally, GIADA and OSIRIS measurements ${ }^{47}$ between 3.6 and 3.4 AU detected 35 outflowing dust grains of mass $10^{-10}$ to $10^{-7} \mathrm{~kg}$ and 48 grains of mass $10^{-5}$ to $10^{-2} \mathrm{~kg}$.

\section{2 $\quad$ First results of in situ measurements}

In situ composition analysis, study of physical-mechanical surface and sub-surface properties: After the first touchdown COSAC and PTOLEMY analyzed the chemical composition of gas and dust close to the surface. COSAC revealed various organic components, including methyl isocyane, acetone, propionaldehyde, and acetone ${ }^{30}$. PTOLEMY detected the main components water vapor, $\mathrm{CO}, \mathrm{CO}_{2}$, and other minor species ${ }^{32}$. The MUPUS thermal probe inserted into the cometary surface by a hammer mechanism at Abydos landing site did not fully penetrate the near-surface layers, indicating the presence of a hard ground material located beneath a $3 \mathrm{~cm}$ thick thin dust layer (local resistance of the ground to penetration larger $4 \mathrm{MPa})^{36}$. The MUPUS infrared radiometer revealed diurnal temperatures between 90 and $130 \mathrm{~K}$, and a local thermal inertia ${ }^{36}$ of $85 \pm 35 \mathrm{~J} \mathrm{~m}^{-2} \mathrm{~K}^{-1} \mathrm{~s}^{-1 / 2}$. The CONSERT radiowave measurements lead to the conclusion that the upper part of 67P's head is homogeneous on a spatial scale of ten meters ${ }^{38}$. Its permittivity results suggest a volumetric dust/ice ratio of 0.4 to 2.6 and a porosity of 75 to $85 \%{ }^{38}$, which is in good correlation with other Rosetta observations.

Close-up in situ imaging: CIVA and ROLIS opened up the possibility to compare two different locations on the cometary surface by close-up in situ imaging ${ }^{50,51}$. The panoramic multispectral camera CIVA imaged the Abydos landing site. These data reveal a fractured surface less covered by dust and therefore perhaps exhibiting more pristine cometary material ${ }^{50}$ (see Fig. 3, right). ROLIS acquired close-range images of the Agilkia ${ }^{51}$ site (see Fig. 3, left) during its descent onto 67P and at the final Abydos landing site (not published yet). The Agilkia and Abydos sites differ considerably. The ROLIS images of Agilkia were taken during Philae's descent. Fig. 3 (left) shows the last descent image a few seconds before touchdown ${ }^{51}$. The ground resolution is $0.95 \mathrm{~cm} /$ pixel disclosing the regolith's texture. Agilkia's surface texture is characterized by a photometrically uniform material composed of debris and blocks ranging in size from centimeters to 5 meters $^{51}$. The surface appears granular (see inset of Fig. 3, left). The presence of aeolian-like features indicates that mobilization and erosion processes take place, which might be driven by airfall-induced deposits ${ }^{51}$. 

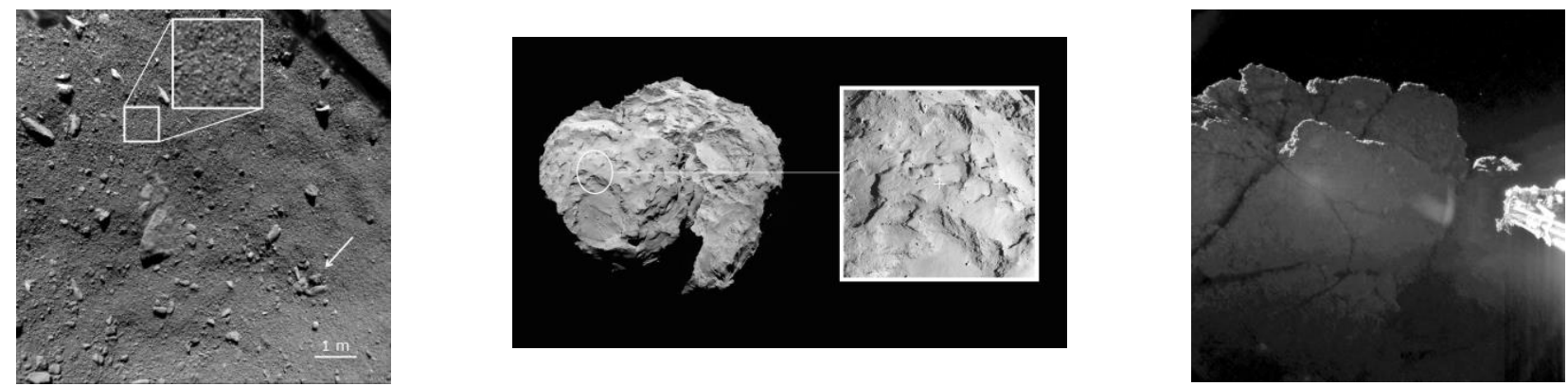

Figure 3. Left: Last image of ROLIS acquired a few seconds before first touchdown (Agilkia area) from an altitude of $9 \mathrm{~m}$ (ground resolution: $0.95 \mathrm{~cm} /$ pixel). The arrow indicates a cluster of blocks ${ }^{51}$.

Middle: Image of the Agilkia site, OSIRIS, credit: ESA/Rosetta/MPS for OSIRIS Team

MPS/UPD/LAM/IAA/SSO/INTA/UPM/DASP/IDA.

Right: CIVA image (Abydos) $)^{50}$.

\section{CONCLUSION AND OUTLOOK}

Rosetta was breaking new ground in many respects. Being the first to land a robotic device on a comet and being the first to orbit and escort a comet, Rosetta provides fundamental data for cometary science. Rosetta's orbiter and in situ measurements suggest that the major mass loss of 67P is driven by sublimation or via removal of material by explosive release from subsurface. Whether 67P's lobes are binary contacted since the early formation 4.6 billion years ago or a single body eroded to form the current shape is still under investigation. The surface morphology displays a high diversity between smooth terrains, large scale depressions, and brittle areas. This diversity is confirmed comparing the different surface textures of Philae's two landing sites. Airfall and surface dust transport form aeolian-like features at the cometary surface. The uppermost surface layer is dark, depleted in volatiles, and rich in complex organic refractories. The low thermal inertia of this material is confirmed by both the orbiter and in situ measurements. Studies of the developing coma enabled to investigate outgassing species and to draw conclusions about the interior composition. As 67P approached its perihelion position in August 2015 it became more and more active enabling to study details of the cometary gases and dust emissions. At the same time the southern hemisphere of $67 \mathrm{P}$ was illuminated progressively disclosing the southern part of the comet. Rosetta's studies of temporal and spatial dynamic processes regarding the nucleus and coma will contribute to understand the sources and effects of cometary activity, while monitoring the nucleus/coma composition will enable to trace the comet's evolutionary path.

\section{ROSETTA TEAM}

Scientific consortia from institutes across Europe and the United States have provided the state-of-the-art scientific instruments. Rosetta's industrial team involves more than 50 contractors from 14 European countries and the United States. The prime spacecraft contractor is Astrium Germany. Major subcontractors are Astrium UK (spacecraft platform), Astrium France (spacecraft avionics) and Alenia Spazio (assembly, integration and verification).

\section{ACKNOWLEDGMENT}

The author acknowledges the revision and comments made by her colleague David Kappel. 


\section{REFERENCES}

[1] Mumma, M.J. and Charnley S. B., "The Chemical Composition of Comets - Emerging Taxonomies and Natal Heritage", Annu. Rev. Astron. Astrophys. 49:471-524, doi: 10.1146/annurev-astro-081309-130811 (2011).

[2] Mumma, M.J. et al., “Organic composition of C/1999 S4 (LINEAR): a comet formed near Jupiter?”, Science 292, 1334-1339 (2001).

[3] Kissel, J. et al., "Composition of comet Halley's dust particles from Giotto observations I', Nature 321, 280-282 (1986a).

[4] Kissel, J. et al., “Composition of comet Halley's dust particles from Giotto observations II”, Nature 321, 336-337 (1986b).

[5] Jessberger, E.K. et al., "Aspects of the major element composition of Halley’s dust", Nature 332, 691-695 (1988).

[6] Barucci, A. et al., "Space missions to small bodies: asteroids and cometary nuclei", Astronomy\&Astrophys. Rev. 19, 48, doi:10.1007/s00159-011-0048-2 (2011).

[7] Sunshine, J.M et al., "Exposed Water Ice Deposits on the Surface of Comet 9P/Tempel 1", Science 311, 1453-1455, doi: 10.1126/science.1123632 (2006).

[8] Li, J.-Y. et al., "Photometric properties of the nucleus of Comet 103P/Hartley 2", Icarus 222, 559-570, doi:10.1016/j.icarus.2012.11.001 (2013).

[9] Schulz, R., “The Rosetta mission- Exploring solar system formation”, Planet. Space Sci. 66, 1 (2012).

${ }^{[10]}$ ESA, Rosetta Factsheet; http://www.esa.int/Our_Activities/Space_Science/Rosetta/Rosetta_factsheet.

${ }^{[11]}$ ESA, Rosetta Orbiter: Instruments: http://www.esa.int/Our_Activities/Space_Science/Rosetta/Orbiter_Instruments.

${ }^{[12]}$ ESA, Rosetta Lander: Experiment: http://www.esa.int/Our_Activities/Space_Science/Rosetta/Lander_Instruments.

${ }^{[13]}$ ESA, Rosetta Overview: http://www.esa.int/Our_Activities/Space Science/Rosetta_overview.

${ }^{[14]}$ Keller, H.-U. et al., "OSIRIS - The scientific camera system onboard Rosetta”, Space Sci. Rev. 128, 433-506, doi:10.1007/s11214-006-9128-4 (2007).

[15] Coradini, A. et al.., "VIRTIS: An imaging spectrometer of the Rosetta mission", Space Science Rev. 128, 529-559 (2007).

[16] Coradini, A. et al., "VIRTIS: An imaging spectrometer for the Rosetta mission", In: Rosetta: ESA's Mission to the Origin of the Solar System, Springer, 565-587 (2009).

[17] Arnold, G. E. et al., "VIRTIS on Rosetta: a unique technique to observe comet 67P/Churyumov-Gerasimenko - first results and prospects", Proc. SPIE, San Diego 2015, paper 9608-2, this issue.

${ }^{[18]}$ Stern, S.A. et al., "Alice-An ultraviolet imaging spectrometer for the Rosetta Orbiter", Adv. Space Res. 21, 15171525, doi:10.1016/S0273-1177(97)00944-7 (1998).

${ }^{[19]}$ Gulkis, S. et al., "MIRO: Microwave instrument for the Rosetta orbiter", Space Sci. Rev. 128, 561-597, doi:10.1007/s11214-006-9032-y (2007).

${ }^{[20]}$ Kofman, Y. et al., "Comet nucleus sounding experiment by radiowave transmission", Adv. Space Res. 21, 15891598, doi:10.1016/s0273-1177(97)00952-6 (1998).

${ }^{[21]}$ Balsinger, H. et al., "ROSINA-Rosetta-Orbiter-spectrometer-for-ion-and-neutral-analysis", Space Sci. Rev. 128, 1-21, doi:10.1051/0004-6361/201424590 (2007).

${ }^{[22]}$ Kissel, J. et al., "COSIMA: High resolution time-of-flight secondary ion mass spectrometer for the analysis of cometary dust particles onboard Rosetta”, In: Schulz, R.; Alexander, C.; Boenhard, H.; Glassmeier, K.H. (Eds.), ESA mission to the origin of the solar system, Springer, New York, 201-242 (2009).

${ }^{[23]}$ Colangeli, L. et al., "The Grain Impact Analyser and Dust Accumulator (GIADA) experiment for Rosetta mission: Design, performances and first results", Space Sci. Rev. 128, 803-821, doi:10.1007/s11214-006-9038-5 (2007).

${ }^{[24]}$ Riedler, W. et al., "The MIDAS experiment for the Rosetta mission", Adv. Space Res. 21, 1547-1556, doi:10.1026/S0273-1177(97)00947-2 (1998).

${ }^{[25]}$ Carr C. et al., "RPC: the Rosetta plasma consortium", Space Sci, Rev. 128, 629-647, doi:10.1007/s11214-006-9136-4 (2007).

${ }^{[26]}$ Pätzold, M. et al, "Rosetta Radio Science Investigations (RSI)", Space Sci, Rev. 128, 599-627, doi:10.1007/s11214006-9117-7 (2007).

[27] Bibring, J.-P. et al., "CIVA”, Space Sci. Rev. 128, 397-412, doi:10.1007/s11214-006-9135-5 (2007).

${ }^{[28]}$ Mottola, S. el al., "The ROLIS experiment on the Rosetta lander", Space Sci. Rev. 128, 241-255, doi:10.1007/s11214-006-9004-2 (2007).

[29] Goesmann, F. et al., "The cometary sampling and composition experiment on Philae", Space Sci. Rev. 128, 257-280, doi:10/1007/s11214-006-9000-6 (2007). 
[30] Goesmann, F. et al., "Organic components on comet 67P/Churyumov-Gerasimenko revealed by COSAC mass spectrometry”, Science 349, aab0689-2, doi:10.1126/science.aab0689 (2015).

[31] Morse, A.D. et al., "Ptolemy - a GCMS to measure the chemical and stable isotopic composition of a comet", In: Schulz, R.; Alexander, C.; Boenhard, H.; Glassmeier, K.H. (Eds.), ESA mission to the origin of the solar system, Springer, New York, 669-689 (2009).

[32] Wright, I.P, et al., "CHO-bearing organic components at the surface of 67P/Churyumov-Gerasimenko revealed by Ptolemy", Science 349, aab0673-1, doi:10.1126/scince.aa0673 (2015).

${ }^{[33]}$ ESA, Rosetta Lander Systems, PTOLEMY: http://sci.esa.int/rosetta/31445-instruments/?fbodylongid=896.

${ }^{[34]}$ ESA, Rosetta Lander Systems, APEX: http://sci.esa.int/rosetta/31445-instruments/?fbodylongid=893.

[35] Spohn, T. et al., "MUPUS-A thermal and mechanical properties probe for the Rosetta lander Philae", Space Sci. Rev. 128, 339-362, doi:10.1007/s11214-006-9081-2 (2007).

${ }^{[36]}$ Spohn, T. et al., "Thermal and mechanical properties of the near-surface layers of comet 67P/ChuryumovGerasimenko", Science 349, aab0464-1, doi:10.1126/science.aa0464 (2015).

[37] ESA, Rosetta Lander Systems, SESAME: http://www.esa.int/Our_Activities/Space_Science/Rosetta/SESAME.

${ }^{[38]}$ Kofman, W. et al., "Properties of the 67P/Churyumov-Gerasimenko interior revealed by CONSERT radar", Science 349, aab0639-5, doi:10.1126/science.aab0639 (2015).

[39] Auster, U. et al., "The nonmagnetic nucleus of comet 67P/Churyumov-Gerasimenko", Science 349, aaa5102-1, doi:10.1126/science.aaa5102 (2015).

${ }^{[40]}$ ESA, Rosetta Lander Systems, SD2: http://www.esa.int/Our_Activities/Space_Science/Rosetta/SD2.

${ }^{[41]}$ Keller, H.U. et al., "Insolation, erosion, and morphology of comet 67P/Churyumov-Gerasimenko", Astronomy\&Astrophysics (2015).

${ }^{[42]}$ Mottola, S. et a., "The rotation state of 67P/Churyumov-Gerasimenko from approach observations with the OSIRIS cameras on Rosetta", Astronomy\&Astrophysics 569, doi:dx.doi.org/10.1051/0004-6361/201424590 (2014).

${ }^{[43]}$ Sierks, H. et al., "On the nucleus structure and activity of comet 67P/Churyumov-Gerasimenko", Science 347, 6220, aaa1044-1 (2015).

[44] Thomas, N. et al., "The morphological diversity of comet 67P/Churyumov-Gerasimenko", Science 347, 6220, aaa044-1 (2015).

${ }^{[45]}$ Gulkis, S. et al., "Subsurface properties and early activity of comet 67P/Churyumov-Gerasimenko", Science 347, aaa0709-1 (2015).

${ }^{[46]}$ Hässig, M. et al., "Time variability and heterogeneity in the coma of 67P/ Churyumov-Gerasimenko", Science 347, aaa0276-1 (2015).

${ }^{[47]}$ Rotundi, A. et al., "Dust measurements in the coma of comet 67P/ Churyumov-Gerasimenko inbound to the Sun", Science 347, aaa3905-1 (2015).

${ }^{[48]}$ Nilsson, H. et al., "Birth of a comet magnetosphere: A spring of water ions", Science 347, aaa0571-1 (2015).

[49] Capaccioni, F. et al., The organic-rich surface of comet 67P/ Churyumov-Gerasimenko as seen by VIRTIS/Rosetta", Science 347 aaa0628-1 (2015).

${ }^{[50]}$ Bibring, J.-P. et al., "67P/Churyumov-Gerasimenko surface properties as derived from CIVA panoramic images", Science 349, aab0671-2, doi: 10.1126/science.aab0671 (2015).

[51] Mottola, S. et al., "The structure of the regolith on 67P/Churyumov-Gerasimenko from ROLIS decent imaging", Science 349, aab0232-1, doi:10.1126/science.aab0232 (2015).

${ }^{[52]}$ ESA, Comet boundaries, 15/07/2015, ESA/Rosetta/MPS for $\quad$ OSIRIS MPS/UPD/LAM/IAA/SSO/INTA/UPM/DASP/IDA: http://www.esa.int/spaceinimages/Images/2015/07/Comet_boundaries_Aten_Aker_Babi_and_Khepry.

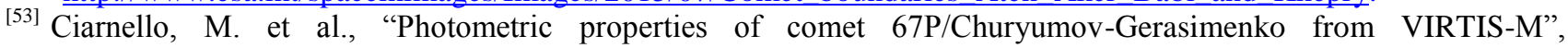
Astronomy\&Astrophys., in press (2015).

[54] Bockelée-Morvan, D. et al., "First observations of $\mathrm{H}_{2} \mathrm{O}$ and $\mathrm{CO}_{2}$ in comet 67P/Churyumov-Gerasimenko with VIRTIS onboard Rosetta”, Astronomy\&Astrophys., in press (2015).

[55] ESA, "Determine mass of comet 67P/C-G: http://blogs.esa.int/rosetta/2014/08/21/determining-the-mass-of-comet$\underline{67 \mathrm{pc}-\mathrm{g} / \text {. }}$ 